\title{
Free space ranging based on a chaotic long-wavelength VCSEL with optical feedback
}

\author{
Ana Quirce ${ }^{\mathrm{a}}$, Pablo Pérez ${ }^{\mathrm{b}, \mathrm{d}}$, Angel Valle*b ${ }^{*}$ Luis Pesquera ${ }^{\mathrm{b}}$, Ignacio Esquivias ${ }^{\mathrm{c}}$, Krassimir \\ Panajotov $^{\mathrm{a}, \mathrm{e}}$, Hugo Thienpont ${ }^{\mathrm{a}}$ \\ ${ }^{a}$ Vrije Universiteit Brussel, Faculty of Engineering Sciences, Brussels Photonics Team B-PHOT, \\ Pleinlaan 2, 1050, Brussels, Belgium; \\ ${ }^{\mathrm{b}}$ Instituto de Física de Cantabria, Consejo Superior de Investigaciones Científicas (CSIC)- \\ Universidad de Cantabria, E-39005 Santander, Spain; \\ ${ }^{\circ}$ Universidad Politécnica de Madrid, CEMDATIC-ETSI de Telecomunicación, Ciudad Universitaria, \\ 28040, Madrid, Spain; \\ dDepartamento de Física Moderna, Universidad de Cantabria, Facultad de Ciencias, E-39005, \\ Santander, Spain; \\ 'Institute of Solid State Physics, 72 Tzarigradsko, Chaussee Blvd., 1784 Sofia, Bulgaria.
}

\begin{abstract}
Chaotic Lidar systems (CLIDAR) are used for high-resolution ranging. They are based on the correlation of a chaotic signal waveform with the signal that is reflected back from the target. We report a novel CLIDAR system based on the autocorrelation of the signal obtained by the superposition of the chaotic signal waveform and the signal that is reflected from the target. A simplified set-up with just one detector is required in contrast to the two detectors used in standard CLIDAR systems. Our experimental results are obtained with a 1550 -nm vertical-cavity surface-emitting laser (VCSEL) with chaotic dynamics due to optical feedback. Our CLIDAR system provides an autocorrelation function with several sharp minima. The position of the target is obtained from the location of those minima. A theoretical analysis of the CLIDAR system is also presented. A rate equation model for the polarization of a VCSEL subject to optical feedback is the basis for the simulation of the CLIDAR system. A comparison between our theoretical and experimental results is performed, resulting in a good agreement in the chaotic signal but in a different sign of the CLIDAR signal.
\end{abstract}

Keywords: Vertical-cavity surface-emitting lasers (VCSELs), optical feedback, chaos, Lidar.

\section{INTRODUCTION}

A broadband chaotic waveform can be generated perturbing a semiconductor laser by an optical feedback, an optoelectronic feedback or an optical injection [1]. This chaotic waveform is used in Chaotic Lidar (CLIDAR) for highresolution ranging [2-4]. A chaotic pulse train from a semiconductor laser can have desirable properties for LIDAR applications due to short pulse width, rapid decorrelation (leading to unambiguous range mesaurements), and high average pulse repetition frequency. In CLIDAR the detection and ranging is realized by correlating the chaotic signal waveform with the time-delayed reference waveform. The resolution of pseudorandom code modulation continuouswave LIDAR is related to the chip length, and therefore of the order of meters for standard systems modulated at tens of $\mathrm{MHz}$ [3], although it can be improved to centimeters via interpolation [5]. CLIDAR has intrinsically higher spatial resolution (of the order of $\mathrm{cm}$ ) benefiting from the wide bandwidth of optical chaos [2-4], which could be further improved through interpolation algorithms. Other advantages of CLIDAR include: there is no ambiguity caused by the limited length of pseudorandom codes or repeated waveforms because a chaotic waveform never repeats itself, and expensive high-speed electronic devices for code generation, amplification and modulation are not needed [2-4].

*valle@ifca.unican.es; phone 34942 201465; fax: 34942200935

Physics and Simulation of Optoelectronic Devices XXIII, edited by Bernd Witzigmann,

Marek Osiński, Fritz Henneberger, Yasuhiko Arakawa, Proc. of SPIE Vol. 9357, 935703

(C) 2015 SPIE · CCC code: 0277-786X/15/\$18 · doi: 10.1117/12.2079508 
In CLIDAR the correlation of the signal and the reference can be carried out either electronically or optically [3]. The performance of CLIDAR is determined by the generated chaotic state. A chaotic source with delta-function autocorrelation function is optimum for ranging applications because it has the highest possible resolution and lowest ambiguity [3]. This delta-function correlated trace can be obtained if the signal is white noise. Alternativelly it can be approached if the signal is chaotic with a large chaotic bandwidth.

Other applications for the CLIDAR technique have been studied recently such as a novel reflectometry method using chaotic signal for the detection of faults on wires [6-7] and a transmitter for underwater ranging and imaging applications [8]. In the ranging technology, the target detection is realized by correlating the reference chaotic signal with the delayed probe signal, and in most of the CLIDAR systems, two detectors are required [2-11]. The chaotic waveform in [3] was generated by using a DFB laser with optical injection. In [3] the signal chaotic bandwidth was $15 \mathrm{GHz}$ and a $3 \mathrm{~cm}$ resolution range was obtained, limited by the detection bandwidth of the oscilloscope. Chaotic signals obtained with laser diodes subject to optical feedback have also been used [4]. Ranging distances of at least $130 \mathrm{~m}$ with a rangeindependent resolution of $18 \mathrm{~cm}$ (limited by the acquisition bandwidth) have been recently obtained [4].

In this work, we propose a novel CLIDAR system based on the autocorrelation of the signal obtained by the superposition of the chaotic signal waveform and the signal that is reflected back from the target. Our chaotic signal is generated using a long-wavelength vertical-cavity surface-emitting laser (VCSEL) subject to optical feedback. The main advantage of our system is that only one detector is required in contrast to the two detectors required in the standard CLIDAR systems. A comparison between our theoretical and experimental results is performed, resulting in a good agreement in the chaotic signal but in a different sign of the CLIDAR signal.

The paper is organized as follows. In section II we describe the experimental setup. Section III is devoted to the presentation of our experimental results. In section IV we present the theoretical model. In section V we describe our theoretical results and compare with our experimental results. Finally, in section V, a discussion and conclusions are presented.

\section{EXPERIMENTAL SETUP}

A schematic of the experimental setup is shown in Fig. 1. A commercial single-mode $1550 \mathrm{~nm}$-VCSEL (Raycan TM) that emits around $1559.3 \mathrm{~nm}$ at $298 \mathrm{~K}$ is used as chaotic source under optical feedback for free space ranging. The bias current and temperature are controlled by a laser driver (Thorlabs LDC200) and a temperature controller (Thorlabs TED200), respectively. The feedback loop is provided by an optical circulator (OC1). The light from the VCSEL passes through the port 2 to the port 3 of the optical circulator OC1.

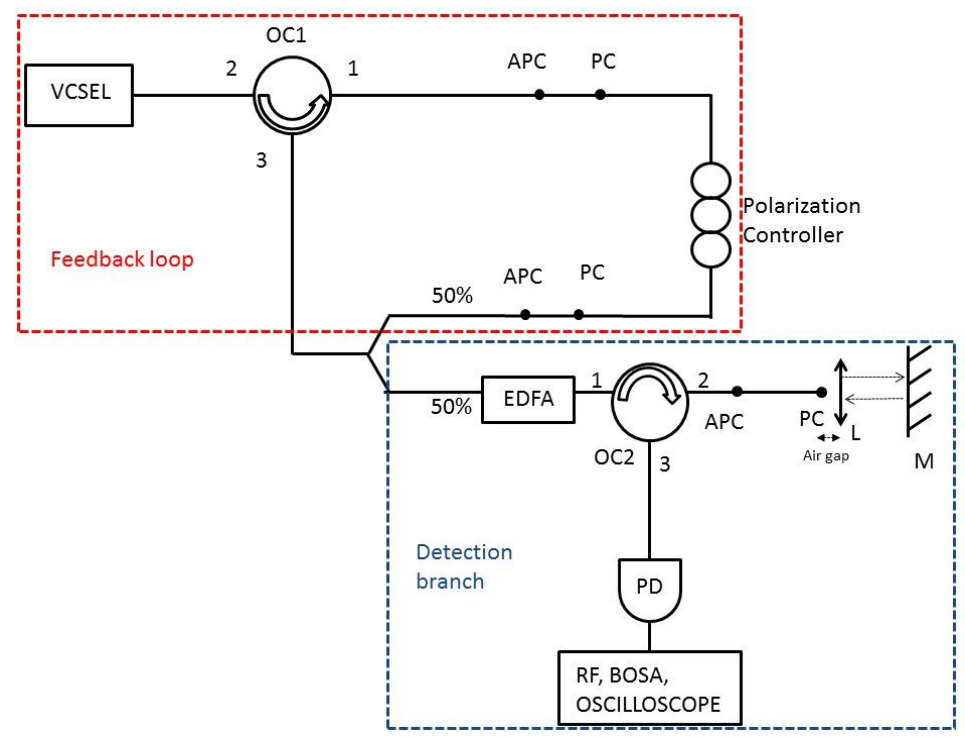

Fig.1. Experimental setup for free space ranging. OC1 and OC2 represent optical circulators; EDFA, Erbium Doped Fibre Amplifier; L, collimating lens; M, mirror; PD, amplified detector; RF, radio-frequency spectrum analyser; BOSA, high-resolution optical spectrum analyser. APC-PC represent different fibre patch cords. 
The light is split in two branches by a 50/50 fibre coupler. One of the branches is connected to an erbium doped fibre amplifier (EDFA) to amplify the chaotic signal obtained by the feedback loop, and the second one to a polarization controller (PC). The PC is used to adjust the polarization of the light re-injected into the VCSEL through the port 1 and the port 2 of the optical circulator OC1 in order to achieve emission of the VCSEL in a chaotic regime. The part of the setup in red colour constitutes the feedback loop.

In the detection branch (in blue colour), the light from the EDFA travels to the port 1 and then to the port 2 of the second optical circulator (OC2).Next, the light is collimated by a collimating lens (L) and it travels to the target mirror (M) propagating through the free space. The signal reflected back from the target is focused by the collimating lens on the PC connector of a FC/PC - FC/APC fibre patch cord. The light at the output of the port 3 of the OC2 is detected with one amplified photodetector ( $9 \mathrm{GHz}$ bandwidth, Thorlabs PDA8GS) and is analysed with a radio-frequency spectrum analyser (Anritsu MS2719B), a high-resolution optical spectrum analyser (Aragon Photonics BOSA 210) with $10 \mathrm{MHz}$ of resolution, or with a $12 \mathrm{GHz}$ bandwidth oscilloscope. Note here, that the fibre patch cords (APC-PC) are used to avoid unwanted reflections between the fibre connectors, except the fibre patch cord next to the lens. In this case, the reflections at the FC/PC connector are used as a reference signal, to determine using the CLIDAR technique, the distance to a target. In all the results presented in this work, the length of the feedback loop is 17.5 meters and the distance between the collimating lens and the mirror is $137 \mathrm{~cm}$.

\section{EXPERIMENTAL RESULTS}

Our VCSEL has a threshold current of $1.6 \mathrm{~mA}$ and a birefringence of $27.24 \mathrm{GHz}$ for a temperature of $298 \mathrm{~K}$. At this temperature and for currents lower than $6 \mathrm{~mA}$, the free-running VCSEL emits in a linear polarization that we will call parallel polarization. When the current is $6 \mathrm{~mA}$, a polarization switching is obtained and the VCSEL emits in the orthogonal polarization. The optical spectrum of the free running VCSEL at $6.47 \mathrm{~mA}$ of bias current and 298K, can be seen in Fig. 2.

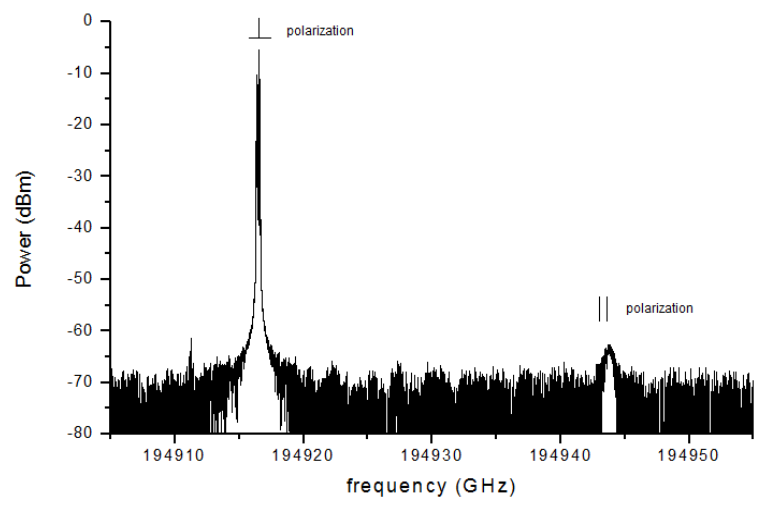

Fig. 2. Optical spectrum for the free-running VCSEL for a bias current of $6.47 \mathrm{~mA}$ and $298 \mathrm{~K}$.

From now on we choose the zero value of the frequency in all the optical spectra to correspond to the orthogonal polarization of the VCSEL. During all the measurements presented in this work, the VCSEL operates at $6.47 \mathrm{~mA}$ of bias current and $298 \mathrm{~K}$. CLIDAR uses optical chaos as the light source. Its performance is mainly determined by the chaotic state chosen. The VCSEL should operate in a chaotic state with a flat, smooth and broad spectrum [2-4]. For characterising the chaotic signal, the detection branch is replaced with a radio-frequency spectrum analyser. Fig. 3a shows in black colour, the chaotic radio-frequency spectrum achieved adjusting the polarization controller in the feedback loop.

Fig. $3 b$ shows the optical spectrum in this chaotic regime obtained when the radio-frequency spectrum analyser is replaced with the BOSA (black trace). Fig. 3 also shows (a) the radio-frequency spectrum and (b) the optical spectrum immediately after the EDFA (red traces). The power measured at the output of the EDFA is $350 \mu \mathrm{W}$. As it is shown in Fig. 3 the chaotic signal has broadband spectra with and without EDFA. The EDFA does not change significatively the chaotic signal of the VCSEL generated by the optical feedback, although the power level is slightly higher than without 
EDFA. The chaos bandwidth of the signal can be defined as the spectral segment accounting for $80 \%$ of the total power in the power spectrum [12]. Using this definition we obtain that the chaos bandwidth is $5.5 \mathrm{GHz}$ and $5.4 \mathrm{GHz}$ for the case without and with EDFA, respectively.

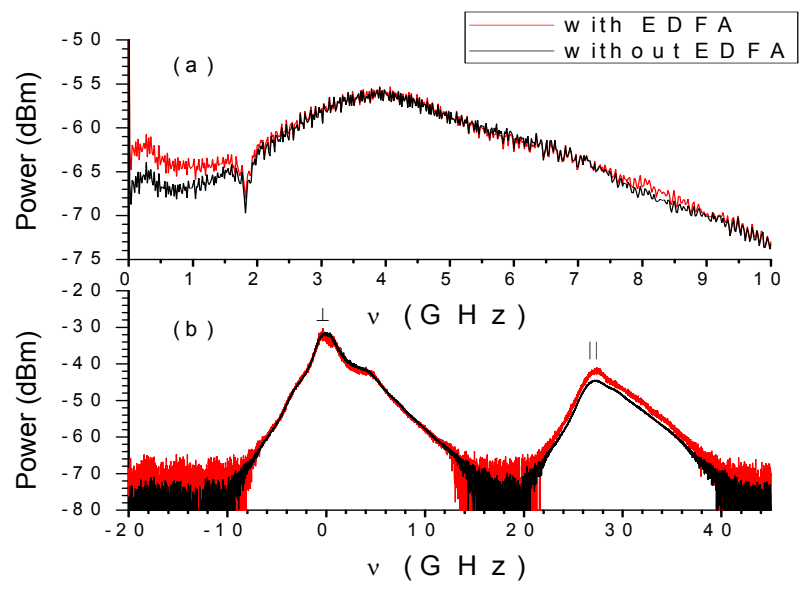

Fig. 3. (a) Radio-frequency spectrum and (b) optical spectrum for the chaotic signal after the feedback loop. Red (Black) colour represents the spectra obtained after (before) the EDFA.

Fig. 4 shows (a) in red (black) colour the autocorrelation trace with (without) EDFA and (b) the time trace for the chaotic signal before the EDFA. Waveforms are displayed and recorded on our digitizing real-time oscilloscope with a 40-GS/s sampling rate. The autocorrelation is carried out with a personal computer. The length of the feedback loop can be determined from the peak position that appears at $85.8 \mathrm{~ns}$ in the autocorrelation trace. This time corresponds to the length of the feedback loop, $17.53 \mathrm{~m}$. The insert in Fig. 4(a) is a zoom of the autocorrelation peak at 85.8 ns. Its Full Width at Half Maximum (FWHM) is 0.09 ns for the case with EDFA.

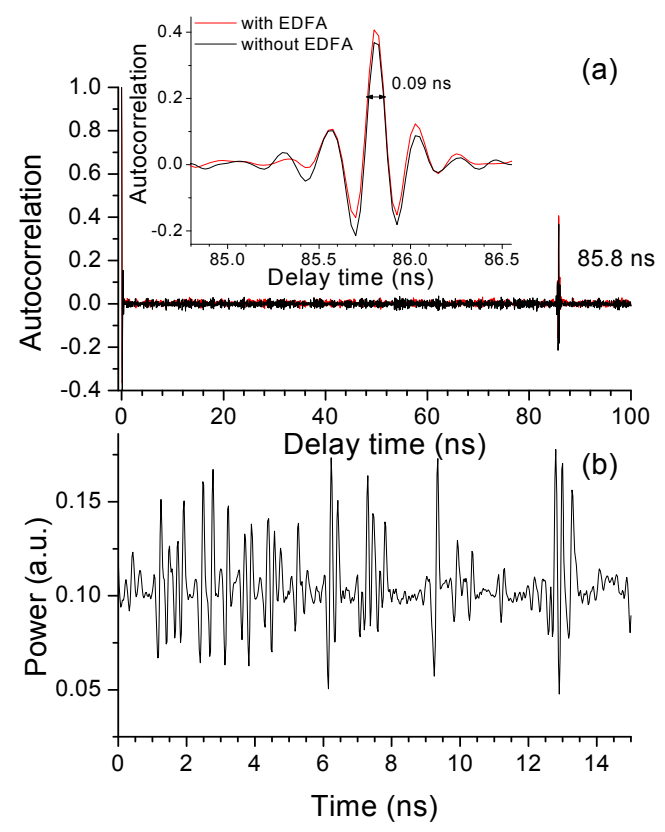

Fig.4. (a) In red (black) colour the autocorrelation trace after (before) EDFA and (b) time series for the chaotic signal before the EDFA. The inset figure in (a) represents a zoom of the peak that appears at $85.8 \mathrm{~ns}$ in the autocorrelation trace. 
Once the chaotic signal is characterized, the light is directed to the target mirror through the detection branch, and the signal observed at the port 3 of the OC2 is analyzed. This signal is a superposition of the light that is reflected at the FCPC connector that is close to the lens and of the light that is reflected at the mirror and is coupled back again in OC2. Fig. $5 \mathrm{a}$ and $5 \mathrm{~b}$ show respectively the optical and radio-frequency spectrum. Frequency oscillations about $0.11 \mathrm{GHz}$ are found in both spectra. This frequency corresponds to the distance between the FC-PC connector and the mirror in a round trip. Besides, the radio-frequency spectrum is flatter and the level of the power has decreased with respect to the radiofrequency spectrum shown in Fig. 3a for the chaotic signal after the EDFA. In this case, the level of the power measured with a power meter at the port 3 of the OC 2 corresponding to the reflected power in the FC-PC connector was $\mathrm{P}_{\mathrm{PC}}=15$ $\mu \mathrm{W}$ (this power has been measured by blocking the free-space beam going to the mirror). However, the light reflected back from the target and coupled into the fibre, measured at the same place, was $\mathrm{P}_{\mathrm{m}}=150.7 \mu \mathrm{W}$.

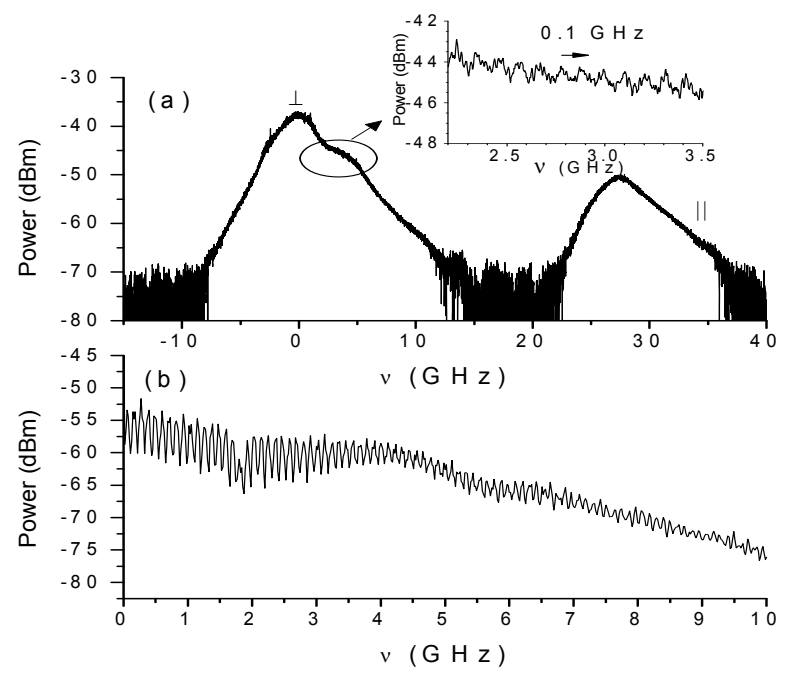

Fig.5. (a) Optical spectrum and (b) radio-frequency spectrum for the ranging results. The inset figure is a zoom of the oscillations present in the optical spectrum. The oscillations that appear in both spectra correspond to a frequency of $0.11 \mathrm{GHz}$ approximately.

The distance between the PC connector and the target can also be obtained from the autocorrelation trace as it is shown in Fig. 6a. We can see in Fig. 6a four peaks in the autocorrelation trace, appart from the peak appearing at zero time delay. An anticorrelation peak appears at $\tau_{\mathrm{m}}=9.1 \mathrm{~ns}$. This peak corresponds to the distance between the PC connector and the target. The main peak that appears at $85.83 \mathrm{~ns}$ corresponds to the length of the feedback loop. Two smaller anticorrelation peaks appear on both sides of the main peak at $76.7 \mathrm{~ns}$ and $94.93 \mathrm{~ns}$ respectively. The distance between these peaks and the main peak also correspond to the distance between the connector and the target, around $9.1 \mathrm{~ns}$. The peaks with negative values of the autocorrelation appear at times related to the distance between connector and mirror. The distance between connector and mirror is $\mathrm{L}=\mathrm{c} \tau_{\mathrm{m}} / 2=137 \mathrm{~cm}$. In the inset figures, we can see that the temporal width of the anticorrelation peak that appears at $9.1 \mathrm{~ns}$ is $\Delta \tau_{\mathrm{m}}=0.22 \mathrm{~ns}$; if this width is considered the worst case spatial resolution as in [3], it corresponds to $\Delta \mathrm{L}=\mathrm{c} \Delta \tau_{\mathrm{m}} / 2=3 \mathrm{~cm}$ for the distance connector-mirror. The distance resolution could be further improved by extracting the peak position via interpolation. Fig. $6 \mathrm{~b}$ shows the time series for the ranging results shown in Fig. 6a. As the time trace shown in Fig. 4b, Fig. 6b also shows the irregular behaviour characteristic of a chaotic regime. As we can see in Fig. 5 and Fig. 6a, the optical spectrum, the radio-frequency spectrum or the autocorrelation trace can be used to determine the distance to the target. We have analysed different ranging results, changing the distance to the target and the amount of power reflected back from the target and coupled into the port 2 of the OC2. We have obtained similar results for smaller distances and higher and smaller values of power coupled, except when the coupled power is small enough $\left(\mathrm{P}_{\mathrm{PC}}=10.9 \mu \mathrm{W}, \mathrm{P}_{\mathrm{PC}}=48.1 \mu \mathrm{W}\right)$ in which just the peaks at $9.1 \mathrm{~ns}$ and $85.8 \mathrm{~ns}$ appear. While the peak at 85.8 is very similar to that shown in Fig. 6a, the anticorrelation observed at $9.1 \mathrm{~ns}$ is much 
weaker (a minimum of -0.1 is obtained). The anticorrelation peak cempletely dissappears if the light reflected back from the target and coupled into the fibre is further decreased (for instance at $\mathrm{P}_{\mathrm{PC}}=11.7 \mu \mathrm{W}, \mathrm{P}_{\mathrm{PC}}=20.2 \mu \mathrm{W}$ ).

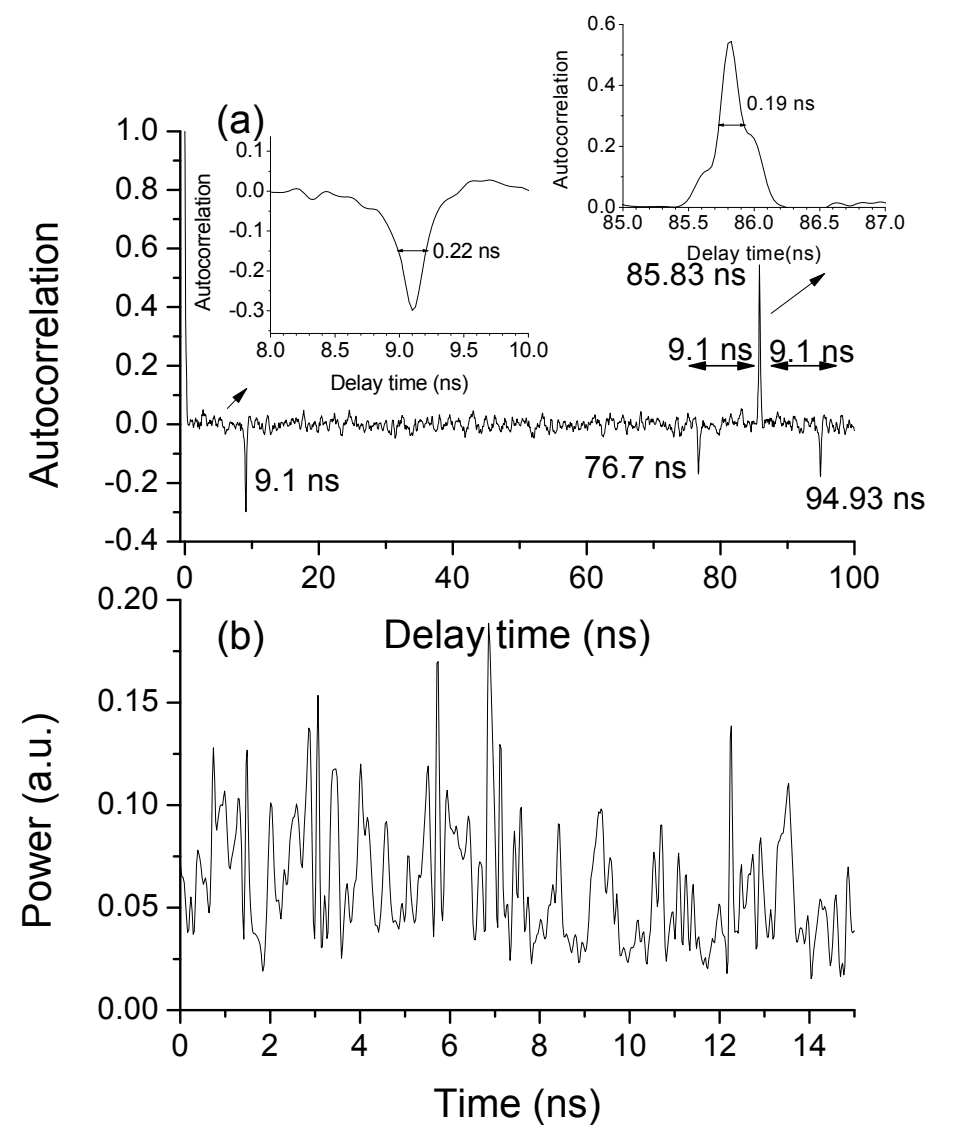

Fig. 6 (a) Autocorrelation trace and (b) time series for the ranging results. The inset figures in (a) show zooms for the peaks that appear at $9.1 \mathrm{~ns}$ and $85.83 \mathrm{~ns}$, respectively.

\section{THEORETICAL MODEL}

VCSELs are a special type of laser diodes with special characteristics in terms of their polarization and transverse mode properties [13]. We focus on a single fundamental transverse mode device with polarization characteristics similar to those observed in [14-15]. In this work we have considered a rate equation model for the polarization of a VCSEL based on the spin-flip model (SFM) [16], in which we introduce a feedback term similar to [17]. We consider an $\mathbf{x}$ (y) direction that is in the plane defined by the VCSEL DBRs that corresponds to the low (high) frequency linear polarization of the VCSEL, that is the orthogonal (parallel) direction analyzed in previous sections. We use the parameters corresponding to a typical single transverse mode VCSEL that were obtained in [14-15]. The model equations are given in (1)-(4), where $E_{x, y}$ are the two linearly polarized slowly varying components of the (scaled) field in the orthogonal $\mathbf{x}$ and $\mathbf{y}$ directions, and $D$ and $n$ are two (scaled) carrier variables. $D$ accounts for the total population inversion between conduction and valence bands, while $n$ is the difference between the population inversions for the spin-up and spin-down radiation channels, and $t$ is the independent variable time. The scaled total population inversion is given by $D=G_{N}\left(N-N_{t}\right) /(2 \kappa)$, where $G_{N}=2.152 \cdot 10^{4} \mathrm{~s}^{-1}$ is the differential gain, $\kappa=33 \mathrm{~ns}^{-1}$ is the field decay rate and $N$ and $N_{t}=9.6 \cdot 10^{6} \mathrm{~s}^{-1}$, are the number of carriers in the active region and at transparency, respectively. The same scaling factor is used for $n$. The rest of internal VCSEL parameters are as follows:, $\alpha=2.8$ is the linewidth enhancement factor, $\mu$ is a normalized bias current, $\gamma_{s}=2100 \mathrm{~ns}^{-1}$ is the spin-flip relaxation rate, $\gamma_{a}=-2 \mathrm{~ns}^{-1}$ is the linear dichroism, $\gamma=2.08 \mathrm{~ns}^{-1}$ is the decay rate of $D$ and $\gamma_{p}=95.19 \mathrm{~ns}^{-1}$ is the linear birefringence. 


$$
\begin{aligned}
\frac{d E_{x}}{d t} & =-\left(\kappa+\gamma_{a}\right) E_{x}-i\left(\kappa \alpha+\gamma_{p}\right) E_{x}+\kappa(1+i \alpha)\left(D E_{x}+i n E_{y}\right) \\
& +\kappa_{f} E_{x}(t-\tau) e^{-i \omega_{x} \tau}+\left(\sqrt{\frac{R_{+}}{2}} \xi_{+}(t)+\sqrt{\frac{R_{-}}{2}} \xi_{-}(t)\right) \\
\frac{d E_{y}}{d t} & =-\left(\kappa-\gamma_{a}\right) E_{y}-i\left(\kappa \alpha-\gamma_{p}\right) E_{y}+\kappa(1+i \alpha)\left(D E_{y}-i n E_{x}\right) \\
& +\kappa_{f} E_{y}(t-\tau) e^{-i \omega_{y} \tau}+i\left(\sqrt{\frac{R_{-}}{2}} \xi_{-}(t)-\sqrt{\frac{R_{+}}{2}} \xi_{+}(t)\right) \\
\frac{d D}{d t} & =-\gamma\left[D\left(1+\left|E_{x}\right|^{2}+\left|E_{y}\right|^{2}\right)-\mu+i n\left(E_{y} E_{x}^{*}-E_{x} E_{y}^{*}\right)\right] \\
\frac{d n}{d t} & =-\gamma_{s} n-\gamma\left[n\left(\left|E_{x}\right|^{2}+\left|E_{y}\right|^{2}\right)+i D\left(E_{y} E_{x}^{*}-E_{x} E_{y}^{*}\right)\right]
\end{aligned}
$$

The scaled spontaneous emission rates are given by $R_{ \pm}=\beta_{S F} \gamma\left[(D \pm n)+G_{N} N_{t} /(2 \kappa)\right]$, where $\beta_{S F}$ accounts for the fraction of spontaneously emitted photons that are coupled into the laser mode. Fluctuations due to spontaneous emission are included in our calculations by $\xi_{+}(t)$ and $\xi_{-}(t)$ (complex Gaussian noise terms of zero mean and time correlation given by $\left.<\xi_{i}(t) \xi_{j}^{*}\left(t^{\prime}\right)>=\delta_{i j} \delta\left(t-t^{\prime}\right)\right)$. In our case, $\beta_{S F}=6.5 \cdot 10^{-4}$. The pumping parameter $\mu$ is related to the bias current, fixed in this work $(I=6.5 \mathrm{~mA})$, the threshold current $\left(I_{t h}=1.602 \mathrm{~mA}\right)$, the number of carriers at transparency $N_{t}$, the number of carries at threshold $\left(N_{t h}=1.21 \cdot 10^{7} \mathrm{~s}^{-1}\right)$, the differential carrier lifetime at threshold $\left(\tau_{e}=1.21 \mathrm{~ns}\right)$ and the carrier lifetime at threshold $\left(\tau_{n}=1 / \gamma=0.48 \mathrm{~ns}\right)$ by

$$
\mu=\frac{\tau_{n}}{\tau_{e}} \frac{\frac{I}{I_{t h}}-1}{1-\frac{N_{t}}{N_{t h}}}+1
$$

Angular frequencies of the $x$ and $y$ polarization, $\omega_{x}$ and $\omega_{y}$, are $\omega_{x}=\alpha \gamma_{a}-\gamma_{p}$ and $\omega_{y}=\gamma_{p}-\alpha \gamma_{a}$. The optical feedback terms are the last ones in equations (1) and (2), where $k_{f}$ is the strength of the feedback, fixed in this work at $8 \mathrm{~ns}^{-1}$ and $\tau$ is the feedback round-trip time, whose value is $83 \mathrm{~ns}$, close to the experimental one.

We have performed several numerical simulations of equations (1)-(5) with an integration time step of $0.01 \mathrm{ps}$ in order to study the characteristics of the VCSEL subject to optical feedback. We consider a situation in which the VCSEL is emitting with a chaotic dynamics in order to measure the distance to a target in a way similar to that considered in the previous section. Optical and radio-frequency spectra are calculated by using a $10 \mathrm{ps}$ sampling time and an average over 1000 temporal traces of $81.92 \mathrm{~ns}$ after a transient of $500 \mathrm{~ns}$. The autocorrelation is calculated by using a $81.92 \mu \mathrm{s}$ time trace with 10 ps sampling time.

\section{THEORETICAL RESULTS}

The chaotic signal that is the basis of our CLIDAR system is characterized by the simulation of equations (1)-(5). Results corresponding to radio-frequency, optical spectrum and autocorrelation function are shown in Fig. 7. Fig. 7a shows the radio-frequency spectrum corresponding to both linear polarizations and to the total power. Also Fig. $7 \mathrm{~b}$ shows the optical spectrum corresponding to both linear polarizations and to the total power. Our theoretical results for the total power describe qualitatively well the corresponding experimental results shown in Fig. 3. Fig. 7a and Fig. 7b show that spectra corresponding to the total power are mainly given by those corresponding to the orthogonal (x) polarization. The main contribution of the y polarization is the broad peak that appears around $30 \mathrm{GHz}$ in the optical spectrum. The chaos 
bandwidth obtained from the noise spectrum of the total power is $5.1 \mathrm{GHz}$, close to the experimental value of section 3 . Also the theoretical autocorrelation function shown in Fig. 7c resembles the corresponding experimental results shown in Fig. 4a. Narrow peaks appear at zero and 83.07 ns delay times, very close to the value of the theoretical feedback roundtrip time. A zoom of the autocorrelation function around the $83.07 \mathrm{~ns}$ value is shown in Fig. $7 \mathrm{~d}$. Our theoretical autocorrelation function has a similar structure to the experimentally observed in the zoom on Fig. 4a. The FWHM in Fig. $7 \mathrm{~d}$ is $0.093 \mathrm{~ns}$, a value close to that experimentally observed in Fig. 4a. Our results are also similar to those obtained with a linearly polarized single-mode quantum-well discrete mode laser diode [18]. It is shown that the autocorrelation function can be approximated by the analytically derived autocorrelation function obtained from a linear stochastic model with delay [18].
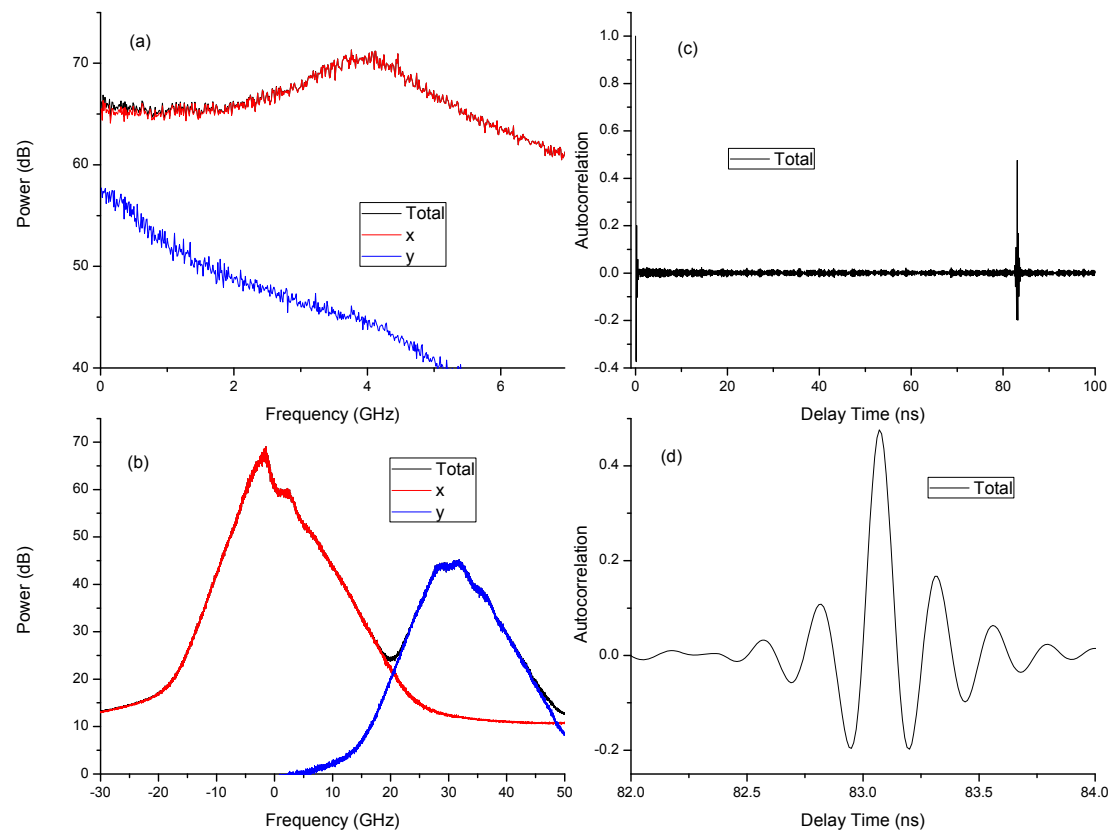

Fig.7. (a) Radio-frequency spectrum, (b) optical spectrum, and (c) autocorrelation function for the VCSEL subject to optical feedback. A zoom of the autocorrelation function in shown in part (d).

We now model the CLIDAR system described in section 2. Our first approximation is to consider that the electrical field is given by the field corresponding to the $x$-polarization, as its contribution to the spectra is dominant (see Fig. 3 and Fig. 7): $\vec{E}(t) \approx E_{x}(t) \vec{i}$. We consider that the time that the light takes to go from the PC connector next to the lens to reflector and to come back again to that PC connector is $\tau_{r}$. The electrical field arriving at the photodetector of Fig. 1 will be proportional to the superposition of the field that is reflected at that PC connector, $a E_{x}(t)$, and of the field that was emitted at that PC connector at time $t-\tau_{r}$, reflected at the mirror and coupled back into the fiber, $b E_{x}\left(t-\tau_{r}\right) e^{-i \omega_{x} \tau_{r}} \cdot a$ and $b$ are constants that depend on the specific components of the feedback loop and detector branch, $\omega_{x}$ is the angular frequency of the $x$-polarized light and $\tau_{r}$ is a parameter that we choose in our simulations (we take $\tau_{r}=9.1 \mathrm{~ns}$ ). Therefore, our simulated CLIDAR signal is

$$
a E_{x}(t)+b E_{x}\left(t-\tau_{r}\right) e^{-i \omega_{x} \tau_{r}}=b\left(\frac{a}{b} E_{x}(t)+E_{x}\left(t-\tau_{r}\right) e^{-i \omega_{x} \tau_{r}}\right)
$$

We show in Fig. 8 the autocorrelation function obtained with Eq. (6) when $a / b=0.23$. Some of the observed experimental features appear also in our simulation results: we find narrow correlation peaks at zero and at $83.1 \mathrm{~ns}$, very close to the feedback time delay, $\tau=83 \mathrm{~ns}$. Also Fig. $8 \mathrm{~b}$ shows that some structure can be seen close to $\tau_{r}$ : a maximum appears 
precisely at $9.1 \mathrm{~ns}$. However in our experiments we have observed a clear anticorrelation peak close to that time that is not observed in our simulations. Also the experimental satellite anticorrelation peaks close to $\tau$ do not appear. Therefore some changes in the model and/or parameters are necessary to describe the experimental results.

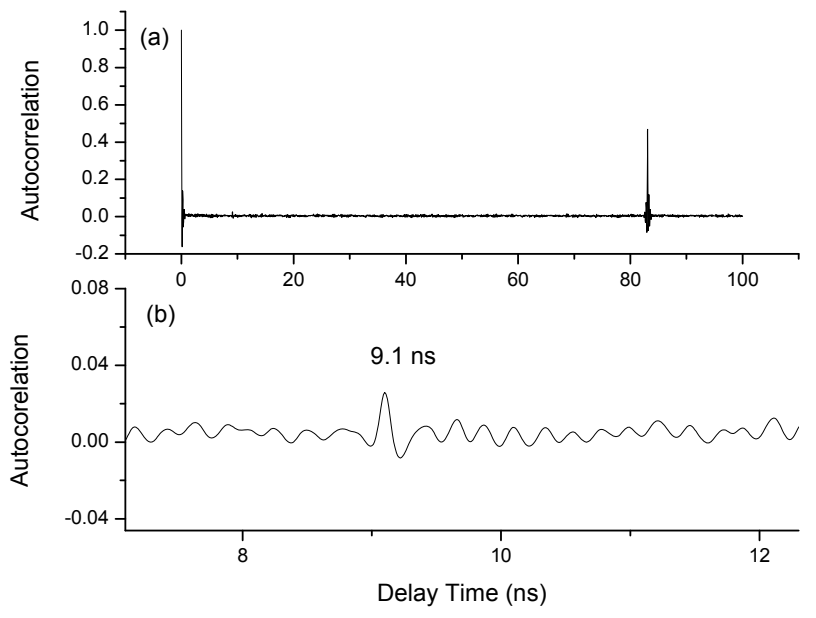

Fig. 8 (a) Theoretical autocorrelation function and (b) corresponding zoom close to $\tau_{r}$ for the CLIDAR signal for $a / b=0.23$

We make a first attempt by changing the $a / b$ factor to 0.8 . We show the results in Fig. 9. Some experimental features are now observed like the satellite peaks at $\tau-\tau_{r}$ and $\tau+\tau_{r}$. However, these peaks, together with the one appearing at $\tau_{r}$ keep on having positive values. Also the background value of the autocorrelation function is not close to zero. These results are not consistent with our experimental results and hence further theoretical work must be done in order to explain succesfully our experimental results.

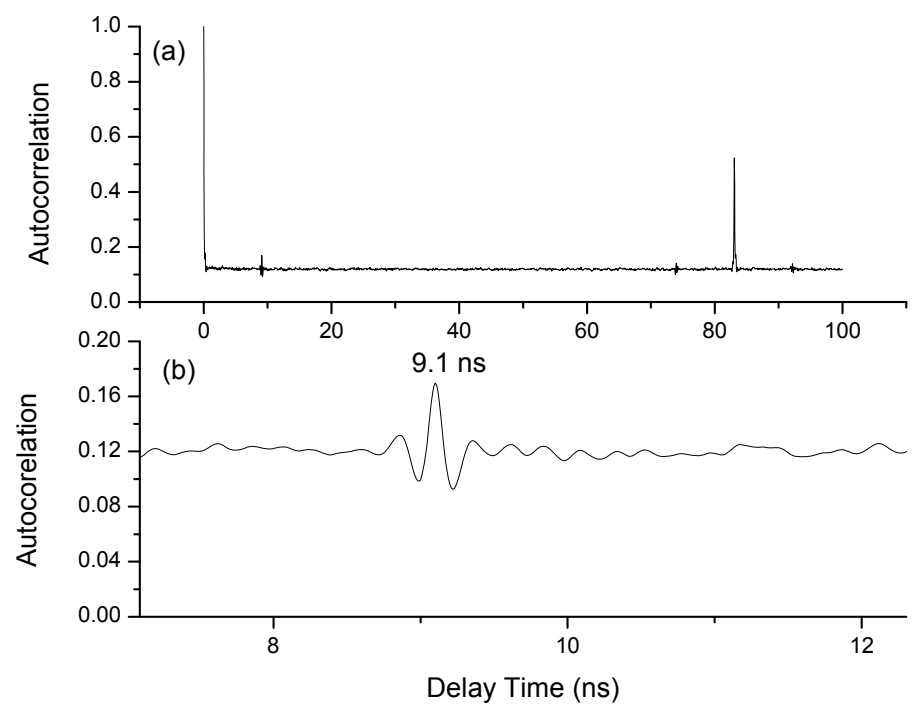

Fig. 9 (a) Theoretical autocorrelation function and (b) corresponding zoom close to $\tau_{r}$ for the CLIDAR signal for $a / b=0.8$

A possible direction in which our theory can be modified in order to obtain more meaningful results is by considering both linear polarizations in the theoretical CLIDAR signal. Fig. 10a shows experimental time traces of the total power of 
the VCSEL with optical feedback in order to compare directly with the theoretical dynamics that is shown in Fig. $10 \mathrm{~b}$. Fig. 10b shows the polarization-resolved dynamical evolution of the VCSEL subject to optical feedback as obtained from Eqs. (1)-(5). We observe that some significative pulses appear in the y-polarization although it is suppressed around $23 \mathrm{~dB}$ in the optical spectrum shown in Fig. 7b. Usually anticorrelation between linear polarizations is observed in the the dynamical evolution of VCSELs [19]. This could be a factor in order to explain the anticorrelation observed in the experimental autocorrelation factor. Further work in this direction is in progress.

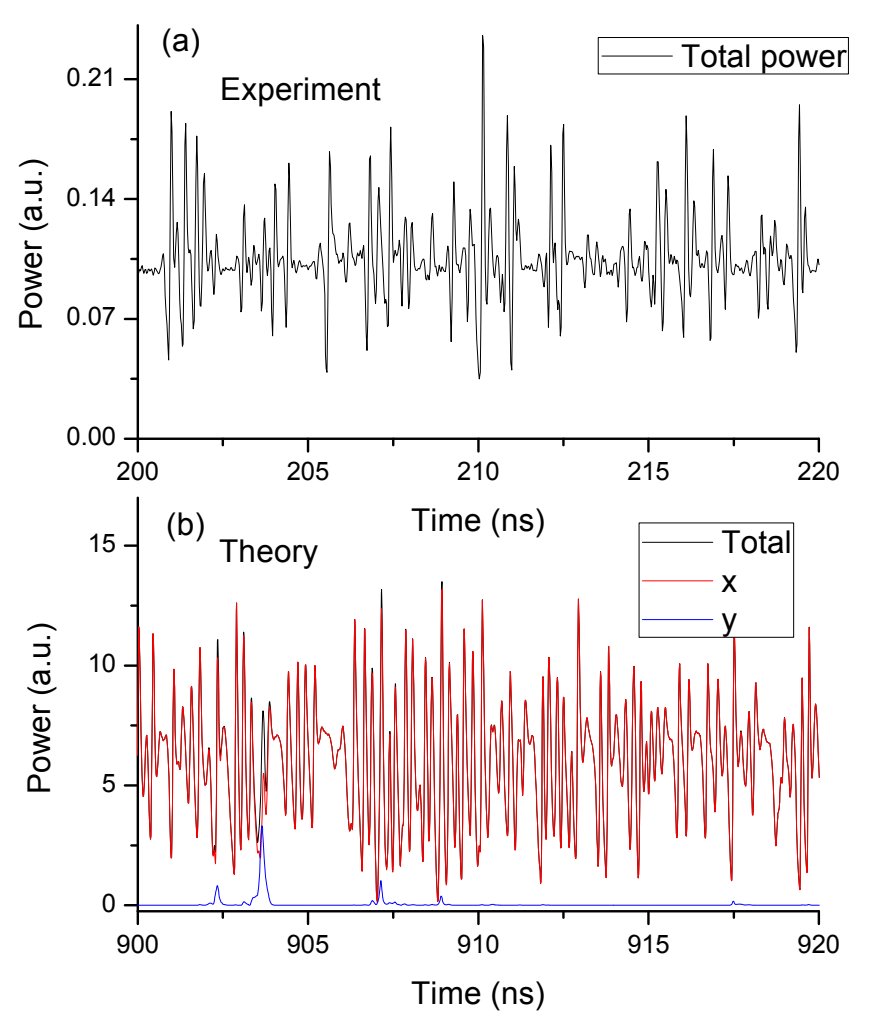

Fig.10. (a) Experimental time series for the total power emitted by the VCSEL before the EDFA. (b) Theoretical time series of the power emitted by each linear polarization of a VCSEL subject to optical feedback.

\section{DISCUSSION AND SUMMARY}

In this work an experimental spatial resolution of $3 \mathrm{~cm}$ has been obtained. This value is similar to other spatial resolutions observed in CLIDAR systems [3-4]. In [3] the resolution is limited by the detection bandwidth of the oscilloscope $(3 \mathrm{GHz})$ while the signal chaotic bandwidth $(15 \mathrm{GHz})$ is not a limiting factor. In our case the resolution is limited by the signal chaotic bandwitdh $(5.5 \mathrm{GHz})$ and not by the detection bandwidth of the oscilloscope. We can expect an improvement of the spatial resolution in our system if bandwidth enhancement of the chaotic signal is obtained. This enhancement can be obtained by using single [20-22] or double optical injection [23] in a VCSEL subject to optical feedback. One characteristic of our CLIDAR system is that the autocorrelation has a peak at the delay time corresponding to the feedback loop that can lead to ambiguity in the determination of the distance. This can be avoided using alternative ways of chaos generation.

Summarizing, we have demonstrated a novel CLIDAR system based on the autocorrelation of the signal obtained by the superposition of the chaotic signal waveform and the signal that is reflected from the target. The main advantage of our 
system with respect to previous CLIDAR system is the use of a single detector, instead of two. A distance to the target of $137 \mathrm{~cm}$ with a spatial resolution of 3 centimeters has been measured. The spatial resolution is comparable to previously reported values in previous CLIDAR systems. A theoretical simulation of our CLIDAR system has also been done. Although comparison between the experimental and theoretical dynamics of the VCSEL subject to optical feedback, that is the input signal of the CLIDAR system, is satisfactory, further work must be done in the theoretical side to explain the anticorrelation peaks observed in the experimental autocorrelation function of the CLIDAR system.

\section{ACKNOWLEDGMENTS}

This work has been funded by the Ministerio de Economía y Competitividad, Spain under project TEC2012-38864-C0303 and cofinanced by FEDER funds. A. Quirce acknowledges FWO for her Post Doc fellowship and H. Thienpont and K. Panajotov are grateful to the Methusalem foundation for financial support.

\section{REFERENCES}

1. J. Ohtsubo, "Semiconductor lasers: stability, instability and chaos," Springer Series in Optical Sciences, Springer 2007.

2. K. Myneni, T. A. Barr, B. R. Reed, S. D. Pethel, and N. J. Corron, "High-precision ranging using a chaotic laser pulse train," Appl. Phys. Lett., vol. 78, no. 11, pp. 1496-1498, 2001.

3. F. Y. Lin, and J. M. Liu, "Chaotic Lidar,” IEEE J. Selec. Top. Quantum Electron., vol. 10, no. 5, pp. 991-997, 2004.

4. T. Zhao, B. Wang, Y. Wang, and X. Chang, "Free space ranging using chaotic light," Mathematical Problems in Engineering, vol. 2013, art. $172728,2013$.

5. X. Ai, R. Nock, J. G. Rarity, and N. Dahnoun, "High-resolution random-modulation cw lidar," Appl. Opt., 50(22), pp. 4478-4488, 2011.

6. Y. Wang, B. Wang, and A. Wang, "Chaotic correlation optical time domain reflectometer utilizing laser diode," IEEE Photon Technol. Lett., vol. 20, no. 19, pp. 1636-1638, 2006.

7. A. Wang, M. Zhang, H. Xu, and Y. Wang, "Location of wire faults using chaotic signal," IEEE Electron Device Letters, vol. 32, no. 3, pp. 372$374,2011$.

8. L. K. Rumbaugh, E. M. Bollt, W. D. Jemison, and Y. Li, "A $532 \mathrm{~nm}$ chaotic Lidar transmitter for high resolution underwater ranging and imaging," IEEE Conference Paper Oceans,San Diego, Sep. 2013.

9. W. T. Wu, Y. H. Liao, and F. Y. Lin, "Noise suppressions in synchronized chaos lidars," Opt. Exp., vol. 18, no. 25, pp. $26155-26162,2010$.

10. Y. Wang, A. Wang, "A novel high resolution chaotic Lidar with optical injection to cahotic laser diode," Proc. of SPIE, vol. 6824, art. 68241I-6, 2007.

11. R. Diaz, S. C. Chan, and J. M. Liu, "Lidar detection using a dual-frequency source," Opt. Lett., vol. 31, no. 24, pp. 3600-3602, 2006.

12. Y. Hong, P. S. Spencer, and K. A. Shore, "Wideband chaos with time-delay concealment in vertical-cavity surface-emitting lasers with optical feedback and injection,” IEEE J. of Quantum Electron., vol. 50, no. 4, pp. 236-242, 2014.

13. R.Michalzik, VCSELs: Fundamentals, Technology, and Applications of Vertical-Cavity Surface-Emitting Lasers. Berlin, Germany, SpringerVerlag, 2012.

14. P. Pérez, A. Valle, I. Noriega, and L. Pesquera, "Measurement of the Intrinsic Parameters of Single-Mode VCSELs," J. of Lightwave Technology, vol. 32, no. 8, pp. 1601-1607, 2014.

15. P. Pérez, A. Valle, and L. Pesquera, "Polarization-resolved characterization of long-wavelength vertical-cavity surface-emitting laser parameters," J. Opt. Soc. Am. B., vol. 31, no. 11, pp. 2574-2580, 2014.

16. J. Martín-Regalado, F. Prati, M. San Miguel, and N. B. Abraham, "Polarization properties of vertical-cavity surface-emitting lasers," IEEE J. Quantum Electron. vol. 33, no. 5, pp. 765-783, 1997.

17. C. Masoller, and N. B. Abraham, "Polarization dynamics in vertical-cavity surface-emitting lasers with optical feedback through a quarter-wave plate," Appl. Phys. Lett., vol. 74, no. 8, 1078, 1999.

18. X. Porte, O. D'Huys, T. Jungling, D. Brunner, M.C. Soriano, and I. Fischer, "Autocorrelation properties of chaotic delay dynamical systems: a study on semiconductor lasers," Phys. Rev. E 90, art. 052911, 2014.

19. A. Valle, M. Sciamanna, and K. Panajotov, "Irregular pulsating polarization dynamics in gain-switched vertical-cavity surface-emitting lasers," IEEE J. Quantum Electron., vol. 44, no. 2, pp. 136-143, 2008.

20. Y. Hong, P. S. Spencer, and K. A. Shore, "Enhancement of chaotic signal bandwidth in vertical-cavity surface-emitting lasers with optical injection," J. Opt. Soc. Am. B, vol. 29, no. 3, pp. 415-419, 2012.

21. Y. Hong, P. S. Spencer, and K. A. Shore, "Flat broadband chaos in vertical-cavity surface-emitting lasers subject to chaotic optical injection," IEEE J. Quantum Electron, vol. 48, no. 12, pp. 1536-1541, 2012.

22. Y. H. Liao and F. Y. Lin, "Dynamical characteristics and their applications of semiconductor lasers subject to both optical injection and optical feedback," Opt. Exp., vol. 21, no. 20, pp. 23568-23578, 2013.

23. M. Zhang, T. Liu, P. Li, A. Wang, J. Zhang, and Y. Wang, "Generation of broadband chaotic laser using dual-wavelength optically injected Fabry-Perot laser diode with optical feedback," IEEE Photon. Technol. Lett, vol. 23, no. 24, pp. 1872-1874, 2011. 